\title{
Note sur la présence de Liriomyza sativae Blanchard au Soudan et au Cameroun (Diptera, Agromyzidae)
}

Michel Martinez, Dominique Bordat

\section{Abstract}

Summary. -The authors wish to report occurrence of Liriomyza sativae in Sudan and Cameroon. This is an extension of the species in the Ethiopian region where, for the first time, serious damages are noted. Informations are given for the geographical distribution, and hostplants for this species as well as for the other harmful Liriomyza species in Africa.

\section{Résumé}

Résumé. -Les auteurs signalent la présence de Liriomyza sativae Blanchard au Soudan et au Cameroun. Ceci correspond à une extension géographique de l'espèce dans la région éthiopienne où, pour la première fois, des dégâts sérieux sont notés. Des informations sont données sur la distribution et les plantes-hôtes de ce ravageur, de même que sur les autres espèces de Liriomyza nuisibles en Afrique.

\section{Citer ce document / Cite this document :}

Martinez Michel, Bordat Dominique. Note sur la présence de Liriomyza sativae Blanchard au Soudan et au Cameroun (Diptera, Agromyzidae). In: Bulletin de la Société entomologique de France, volume 101 (1), avril 1996. pp. 71-73;

https://www.persee.fr/doc/bsef_0037-928x_1996_num_101_1_17218

\section{Ressources associées :}

Liriomyza sativae

Diptera

Agromyzidae

Fichier pdf généré le 24/09/2019 


\title{
Note sur la présence de Liriomyza sativae Blanchard au Soudan et au Cameroun (Diptera, Agromyzidae)
}

\author{
par Michel MARTINEZ * \& Dominique BORDAT**
}

*I.N.R.A., U.F.R. d'Ecologie animale et de Zoologie agricole, 2 place Pierre Viala, F - 34060 Montpellier Cedex 1

** C.I.R.A.D./F.L.H.O.R., 2466, avenue du Val de Montferrand, B.P. 5035, F - 34032 Montpellier Cedex 1

\begin{abstract}
Résumé. - Les auteurs signalent la présence de Liriomyza sativae Blanchard au Soudan et au Cameroun. Ceci correspond à une extension géographique de l'espèce dans la région éthiopienne où, pour la première fois, des dégâts sérieux sont notés. Des informations sont données sur la distribution et les plantes-hôtes de ce ravageur, de même que sur les autres espèces de Liriomyza nuisibles en Afrique.
\end{abstract}

Summary. - The authors wish to report occurrence of Liriomyza sativae in Sudan and Cameroon. This is an extension of the species in the Ethiopian region where, for the first time, serious damages are noted. Informations are given for the geographical distribution, and hostplants for this species as well as for the other harmful Liriomyza species in Africa.

Mots clés. - Diptera, Agromyzidae, Liriomyza sativae, Liriomyza spp. ravageurs, distribution géographique, région éthiopienne.

Parmi les Diptères Agromyzidae, Liriomyza sativae Blanchard est une des espèces mineuses de feuilles les plus polyphages et les plus nuisibles. Elle est répertoriée sur des centaines d'espèces végétales appartenant à 125 genres et 32 familles; les Cucurbitaceae, les Fabaceae (Leguminosae), les Malvaceae et les Solanaceae sont notamment attaquées.

$\mathrm{Sa}$ distribution est relativement vaste puisqu'elle est connue des régions biogéographiques suivantes:

- Néotropicale: elle est largement répandue en Amérique du sud, d'où elle est originaire, ainsi qu'en Amérique centrale et dans les Caraibes.

- Néarctique : elle est présente à l'extérieur dans le sud et l'ouest des Etats-Unis d'Amérique, au nord comme dans l'Ontario (Canada), elle se maintient surtout en serre.

- Australienne/pacifique: elle est fréquente, en particulier dans les îles.

- Orientale: ce ravageur a été récemment signalé de l'Inde et de Thaïlande (MARTINEZ, 1994).

- Paléarctique: la seule citation pour cette région est celle d'Oman, dans la péninsule arabique (DEEMING, 1992).

Dans la région éthiopienne (=afrotropicale), Liriomyza sativae a été recensé au Yémen (DEEMING, 1992), suite à la capture de deux mâles au piège lumineux, et au Zimbabwe (Rhodésie). Concernant cette dernière citation qui apparaît dans divers documents émanant de l'O.E.P.P./E.P.P.O., il nous a été indiqué que cette espèce a été trouvée, en 1991, sur un lot de fleurs coupées en provenance du Zimbabwe, par le Service finlandais de la Protection des Végétaux. Par ailleurs, le Secrétariat de l'O.E.P.P. a effectué, en 1992, une validation des informations sur la répartition géographique des organismes nuisibles (dont faisait partie L. sativae). Le Zimbabwe avait confirmé, à l'époque, la présence de $L$. sativae sur son territoire, dans la catégorie «présent, répartition limitée». Il n'a pas été possible cependant de savoir quel diptériste avait identifié tout d'abord l'espèce du Zimbabwe.

Une mission effectuée au Soudan, du 6 au 17.XII.1995, par D. Bordat a permis de 
constater la présence de cette mineuse dans ce pays où les dégâts étaient sérieux. En janvier 1996, Mlle Faiza Elgaili H. Salah (Université de Gezira à Wad Medani) nous a envoyé d'autres échantillons soudanais récoltés dans plusieurs localités et sur diverses plantes. Dans ce pays, Liriomyza sativae semble bien acclimaté, surtout dans les zones de cultures irriguées ou pluviales autour du Nil blanc et du Nil bleu). Parmi les principales localités où l'espèce a été trouvée, on peut citer: Damazin, El Hawata, El Obeid, Fadasi, Kosti, Sennar, Singa et Wad Medani. Des adultes ont été obtenus en grande quantité à partir de mines prélevées sur diverses plantes cultivées: Hibiscus esculentus L. (Gombo), Lycopersicon esculentum Miller (Tomate), Solanum melongena L. (Aubergine), Citrulus lanatus (Thunb.) (Pastèque), Cucumis melo L. (Melon), Phaseolus vulgaris L. (Haricot) et Ricinus communis L. (Ricin). Les dégâts ont été particulièrement graves surtout sur les jeunes plantes, aussi bien sur les cotylédons que sur les feuilles. Selon les renseignements pris sur place, il semblerait que de tels dégâts soient perpétrés depuis 2 ou 3 ans, ce qui laisse supposer que l'espèce est présente dans ce pays depuis 1993 ou 1994. Il ne fait donc aucun doute qu'elle y est bien établie. Notons que dans plusieurs localités $L$. trifolii (Burgess) se trouve mélangée à $L$. sativae, ces deux espèces exploitant souvent les mêmes plantes-hôtes. La présence au Soudan de $L$. trifolii est plus ancienne que celle de $L$. sativae; en effet, dès 1989, l'un de nous (M.M.) avait identifié des adultes de $L$. trifolii.

Outre le Soudan, nous avons également déterminé des spécimens de L. sativae, provenant du Cameroun (Maroua). Ces derniers ont été obtenus par M. J.-P. Deguine (C.I.R.A.D /C.A.), fin mai 1995, de mines sur feuilles de cotonniers situés à proximité d'habitations. Cependant, à ce jour, il n'a pas été constaté de dégâts en culture de cette mineuse, dans ce pays.

Dans des prélèvement effectués au Soudan nous avons observé la présence de quelques Hyménoptères parasitoïdes des genres Opius (Braconidae), Disorygma (Eucoilidae) et Cirrospilus (Eulophidae).

On peut penser que Liriomyza sativae a colonisé le Soudan à partir de populations provenant du Yémen ou d'Oman. Les vents d'ouest sont fréquents dans ces régions. Dans cette hypothèse, il est probable que l'Ethiopie soit aussi envahie par cette mineuse. Par contre, on s'explique moins bien, pour le moment, la présence de Liriomyza sativae au Cameroun et au Zimbabwe.

Sur les 355 espèces que compte le genre Liriomyza, seulement seize sont signalées de la région éthiopienne. Parmi ces dernières, cinq présentent une importance agronomique en tant que ravageurs. Il s'agit de $L$. brassicae (Riley), L. huidobrensis (Blanchard), $L$. congesta (Becker), L. sativae Blanchard et L. trifolii (Burgess) ${ }^{1}$.

D'après les informations dont nous disposons, ces espèces se sont, pour le moment, diversement établies dans la région éthiopienne. Il nous a paru utile, compte tenu de leur importance agronomique et économique, de donner pour chacune d'entre elles des éléments sur leur distribution géographique, particulièrement afrotropicale, et sur leurs plantes-hôtes.

Liriomyza brassicae. Cette mineuse de feuille a une vaste distribution géographique puisqu'elle est signalée des régions paléarctique, néarctique, néotropicale, orientale, australienne/pacifique et éthiopienne. Dans cette dernière région, elle est connue d'Afrique du Sud, d'Ethiopie, du Kenya, du Mozambique, du Sénégal, du Zimbabwe, des îles du Cap-Vert et de la Réunion! Elle se développe aux dépens d'une dizaine de genres

1 Divers auteurs, dont RISBEC (1950: 277-278) citent Liriomyza strigata (Meigen) d'Afrique. Il s'agit là de citations erronées, suite à des erreurs d'identifications, et nous ne savons pas à quelle(s) espèce(s) elles se rapportent. Liriomyza strigata est confirmé seulement de la région paléarctique. 
de Brassicaceae (surtout les Brassica spp.) et de quelques espèces végétales appartenant principalement aux Capparidaceae (genres Cleome et Gynandropsis) et aux Tropaeolaceae (Tropaeolum majus L.).

Liriomyza congesta. Cette espèce, connue d'Europe, du nord de l'Afrique et de la région orientale est, pour la région éthiopienne, uniquement signalée au Yémen (DEEMING, 1992). Il s'agit d'une mineuse de feuille oligophage, recensée sur 27 genres de Fabaceae. La luzerne (Medicago sativa L.) est fréquemment attaquée.

Liriomyza huidobrensis. Cette espèce est d'origine néotropicale mais elle a été, à la fin des années 1980, accidentellement introduite dans les régions paléarctique, orientale et éthiopienne. Elle est connue aussi des régions néarctique et australienne/pacifique (Guam). Dans la région éthiopienne, elle n'est répertoriée que dans des îles: Mayotte ! ${ }^{2}$, la Réunion! et les Seychelles! Comme $L$. sativae et $L$. trifolii, il s'agit d'une mineuse de feuille très polyphage, elle se développe aux dépens de 33 familles et 108 genres botaniques.

Liriomyza trifolii. Cette espèce, d'origine américaine, est signalée de nombreux pays d'Amérique du sud ainsi qu'aux U.S.A. et au Canada. Elle se trouve également dans la région australienne/pacifique. Elle a été accidentellement introduite dans les régions paléarctique, orientale et éthiopienne. Dans cette dernière région elle est recensée: d'Afrique du Sud !, du Bénin !, de Côte d'Ivoire !, d'Ethiopie !, de la République de Guinée !, du Kenya !, de Madagascar !, de l'Ile Maurice !, de Mayotte !, de la Réunion !, du Sénégal !, du Soudan !, de Tanzanie, de Zambie ! et du Zimbabwe. En raison de son extrême polyphagie, elle se développe aux dépens de 156 genres botaniques appartenant à 41 familles. cette espèce est un ravageur de tout premier plan.

L'identification des cinq espèces de Liriomyza précitées est difficile et nécessite obligatoirement l'examen des organes génitaux des mâles. Elle doit donc être réalisée avec le plus grand soin, dans le cadre d'études ou de recherches qui pourraient être conduites, suite, en particulier, à l'extension de Liriomyza sativae sur le continent Africain et à la menace que représente ce ravageur. Il est probable que cette espèce ne s'acclimate pas de la même façon dans toute l'Afrique, selon qu'il s'agisse de zones sahéliennes, subtropicales ou tropicales. Il est donc hasardeux de tirer, pour cette espèce, des conclusions hâtives quant à son acclimatation totale ou partielle dans tels ou tels pays, zones ou régions.

REMERCIEMENTS. - Nous remercions vivement le secrétariat de I'O.E.P.P. pour les informations communiquées à propos de la présence de $L$. sativae au Zimbabwe, Mlle Faiza Elgaili $H$. Salah et M. JeanPhilippe Deguine pour le matériel qu'ils nous ont transmis, respectivement du Soudan et du Cameroun.

\section{AUTEURS CITÉS}

DeEming J.C., 1992. - Liriomyza sativae Blanchard (Diptera: Agromyzidae) established in the Old World. Tropical Pest Management, 38 (2) :218-219.

MARTINEZ M., 1994. - Un nouveau ravageur menace la région orientale : Liriomyza sativae Blanchard (Diptera, Agromyzidae). Bulletin de la Société entomologique de France, 99 (4) : 356.

RisBEC J., 1950. - I, La faune entomologique des cultures au Sénégal et au Soudan français. II, Contribution à l'étude des Proctotrupidae. Travaux du Laboratoire d'Entomologie du Secteur Soudanais de Recherches Agronomiques. Station expérimentale de M'Bambey (Sénégal) et Section Technique d'Agriculture Tropicale de la France d'Outre-Mer, 639 pp.

2 ! = donnée vue et confirmée par l'auteur. 